\title{
Growth Response of Neurofibroma Explants to FGF-Basic Human Single-Plex Beads In -Vitro Cell-Culture Analysis
}

\author{
Sulaiman $\mathrm{MH}^{1}$, Imam J11, Suleiman $\mathrm{MM}^{2}$, Bello $\mathrm{A}^{3 *}$, and Kudi CA4 \\ 1Department of Veterinary Medicine, Ahmadu Bello University Zaria, Nigeria \\ 2Department of Veterinary Physiology and Toxicology, Ahmadu Bello University Zaria, \\ Nigeria \\ ${ }^{3}$ Department of Veterinary Anatomy, Usmanu Danfodio University Sokoto, Nigeria \\ ${ }^{4}$ Department of Veterinary Medicine, Ahmadu Bello University Zaria, Nigeria
}

\section{Research Article \\ Volume 1 Issue 2}

Received Date: January 06, 2017

Published Date: January 20, 2017

*Corresponding author: AbdulRahman Bello, Department of Veterinary Medicine, Ahmadu Bello University Zaria, Nigeria, Tel: +2348162950036; E-mail: abccrcfge28@gmail.com

\section{Abstract}

Neurofibromatosis type 1 (NF-1), also known as von Recklinghausen disease is caused by disorder of a single gene on chromosome 17 that usually restrains cell division. The NF1 gene is mapped to chromosome 17(17p21). Its protein product neurofibromin, negatively regulate oncogenic Ras, and thus regulate cell proliferation. Neurofibromas are heterogeneous and complex benign tumours, consisting of Schwann cells (NF-/-) and fibroblasts (NF1+/+, NF+/-) and other cell types including perineurial cells, mast cells, pericytes and endothelial cells. Human basic fibroblasts growth factor (b-FGF) is expressed by Schwann cells, macrophages and fibroblasts at the distal nerve stump, thereby preparing the local environment for axonal regeneration. Thus, b-FGF is apparently imperative in Schwann cell proliferation. This study was undertaken in order to elucidate any growth advantage conferred on benign neurofibroma explants following the introduction of b-FGF. Neurofibroma specimens from 22 patients were processed in accordance to the standard operating procedure of primary cell culture laboratory. Cell culture analysis suggests an increased in cell proliferation as positive response to b-FGF in cell phenotypes with NF1-/+. Conversely, cell phenotypes with NF1+/+ did not respond to the addition of (b-FGF) in terms of cell proliferation. Indeed, the cells with NF1+/+ phenotypes exhibited the features reminiscence of cell arrest and / senescence. It is hypothesize herein, that cells with haploinsufficient NF1 gene (NF1+/-) and those with null genotypes (NF1-/-) may be responsible for the proliferation of the neurofibroma in vivo and thus can be utilize as critical targets for therapeutic management of neurofibromatosis type- 1 .

Keywords: NF1; Basic FGF; Haploinsufficient NF1 gene; Schwann cells; Fibroblasts; Cell culture

\section{Introduction}

Neurofibromatosis type 1 (NF1) is one of the common autosomal disorders in human with an estimated birth incidence of 1 in 3500 people worldwide. NF1 affects virtually all body tissues and organs [1]. Neurofibromas are heterogeneous and complex benign tumours consisting mostly of Schwann cells and fibroblasts and 
other cell types such as axons, perineurial cells, mast cells, pericytes, endothelial cells, smooth muscle cells, collagen and cells with intermediate features [1,2]. Schwann cells are reported to constitute the major cell type (40-80\%) in neurofibroma as defined by positive staining for the $\mathrm{S}$ $100 \beta$ protein [1]. In healthy adult peripheral nerve, Schwann cells are believed to be generally quiescent. Similarly, cultures of isolated rat sciatic nerve Schwann cells hardly proliferate in serum-supplemented medium [3]. The possibility that Schwann cells negatively regulate their own proliferation was supported by the demonstration that conditioned media from Schwann cell cultures inhibited the proliferation of mitogen-stimulated test cultures.

The inhibition could be "complete, dose dependent" and exhibited when the test Schwann cells were under the influence of different types of mitogens such as cholera toxin, laminin, and living neurons [3]. The inhibition of proliferation was completely reversible and a rapid doubling of cell number resulted when treatment with conditioned medium was withdrawn from mitogenstimulated Schwann cells. The results suggest that Schwann cells produce an autocrine factor, designated as a "neural antiproliferative protein," which completely inhibits the in vitro proliferation of Schwann cells but not that of immortalized Schwann cells such as neurofibroma cells or Schwannoma lines [3].

Human Schwann cells and cutaneous neurofibroma Schwann cell cultures secreted abundant matrix metalloproteinase -3 (MMP-3), and their proliferation was inhibited by autologous and rat Schwann cell conditioned media [4]. However, neurofibroma Schwann cells were highly invasive and secreted up to 10-fold more MMP-2 than normal human Schwann cells [4]. Additionally, only cutaneous neurofibroma Schwann cell cultures secreted MMP-9 (gelatinase B) and MMP-1 (interstitial collagenase) and also invaded the native type I collagen barriers [4]. Cultures of normal Schwann cells and plexiform neurofibroma tumour expressed little or no MMP-1 and did not invade type I collagen barriers [4]. These results suggest a role for MMPs in the control of proliferation and invasion by Schwann cells and in the formation of peripheral nerve sheath tumours. Wu et al., reported that the embryonic mouse NF1 (-/-) Schwann cells exhibit increased proliferation and less growth factor-dependence in vitro compared with heterozygous and wild-type counterparts. Moreover, NF1 (-/-) Schwann cells showed tomourigenic growth when implanted into nerve of adult NF1 heterozygous mice [5]. These findings support the conclusion that loss of NF1 in embryonic mouse Schwann cells is sufficient for tumour development in the heterozygous environment of adult mouse nerve.

Similarly, plexiform neurofibromas are slow-growing benign tumours that are highly vascular and can progress to malignancy. The development of neurofibromas requires loss of both NF1 alleles in Schwann cells destined to become neoplastic, and may be exacerbated by NF1 heterozygosity in other non-neoplastic cells. $\mathrm{Wu}$ et al., reported that NF1 heterozygous mice showed increased neovascularization in both the retina and cornea in response to hypoxia and basic fibroblast growth factor (bFGF) respectively, compared to their wild-type litter mates [6]. The increase in corneal neovascularization was associated with heightened endothelial cell proliferation and migration, and increased infiltration of inflammatory cells. In addition, NF1 heterozygous endothelial cell cultures showed an exaggerated proliferative response to angiogenic factors, particularly to bFGF [6]. These findings support the conclusion that NF1 heterozygosity in endothelial cells and perhaps inflammatory cells augments angiogenesis, which may promote neurofibroma formation.

Highly-enriched Schwann cell (SC) cultures were established from 10 dermal and 8 plexiform neurofibromas by selective subculture using glial growth factor-2 and laminin. These cultures had delayed senescence, a lack of density-limited growth and a strong propensity to spontaneously form proliferative cell aggregates, rich in extracellular matrix [7]. Thus, indicating that neurofibromin-deficient SC had a substantial growth advantage. Immunohistochemical staining of the originating tumours showed that majority comprised principally neurofibromin-negative SCs, while the remainder contained both neurofibromin-negative and neurofibromin-positive SCs [7]. Importantly, the engraftment of neurofibromin-deficient SCs cultures into the peripheral nerves of mice consistently produced persistent neurofibroma-like, with diffuse and often extensive, intraneural growth [7]. Consequently, it is proposed that these observations indicate that neurofibromin-deficient SCs are involved in neurofibromas formation.

The culture of SCs from the normal peripheral nerve and benign neurofibroma presents a unique challenge because the cell explants from the peripheral nerves and the neurofibroma tissue biopsies are very heterogeneous, 
containing a large number of fibroblastic cells in addition to the SCs. Thus we carried out a standard in vitro analysis of the growth pattern of benign familial neurofibroma, plexiform and sporadic neurofibromas with and without b-FGF. The normal human peripheral nerves served as a comparative tissue and were subjected to the same procedure as the tumours.

Little evidence exists in the literature that described the behaviour in vitro of both the heterozygous NF-1 fibroblast cells (NF1+/-) and the Schwann cells that harbour the mutant alleles (NF1-/-) in cell cultures of different neurofibromas. All previous works were conducted on mouse embryonic SCs. Thus, this study provides for the first time, an insight into the behavioural pattern of cell growth of both the fibroblastic cells and the SCs, derived from clinically-diagnosed human neurofibroma. The response of the normal peripheral nerve to growth factor stimulus was also assessed and compared with those of the benign neurofibromas. Therefore, in addition to providing the vital information on cell growth pattern of the benign neurofibromas, strategic therapeutic intervention could be tailored towards the growth pattern of the tumour cells, and thus providing an effective clinical management of NF1.

\section{Materials and Methods}

\section{Tissues}

A total of 19 benign neurofibromas comprising 7 familial, 6 plexiform and 6 sporadic neurofibroma tissues were utilized for this study. Eight (8) normal peripheral nerves, obtained from amputation stumps were used as comparative and control tissues. After obtaining informed consent 48 hours before surgery from patients due to undergo surgery at the Liverpool and Broadgreen Royal Hospitals UK, with accompanying ethical approval $(06 / 1505 / 137)$, the resected tissues from 19 patients were placed in a container and transported on ice. Subsequently, the tumour specimens were washed with sterile phosphate -buffered saline solution (PBS) with $\mathrm{pH}$ 7.6 in a Petri dish. Tumour specimens were minced into 1$\mathrm{mm}^{3}$ pieces and incubated with $0.1 \%$ trypsin (Zymed) and $0.05 \%$ collagenase type 1 (Zymed) at $37^{\circ} \mathrm{C}$ in Dulbecco modified eagle meduim (DMEM) with $1 \%$ penicillin and streptomycin and $10 \%$ foetal calf serum overnight (12-18 hours) in a $25 \mathrm{~cm}^{2}$ culture flask in order to aid dissociation. Specimens were examined the following day for evidence of dissociation. Once the dissociation was evident, the specimens were transferred to centrifuge tubes and centrifuged at 1200 gravity (g) for 2 minutes.
The supernatant was discarded and the cell pellets washed twice with PBS. This procedure was repeated three times. Finally, the dissociated cells and the explants were resuspended in DMEM with $1 \%$ penicillin and streptomycin. Cultures were grown at $37^{\circ} \mathrm{C}$ with $95 \% \mathrm{O}_{2}$, $5 \% \mathrm{CO}_{2}$, and $100 \%$ humidity. Cultures were constantly monitored for growth and contamination with the aid of an inverted light microscope.

The media were first changed at the third day of the culture, and then every other day thereafter. Following $70 \%$ confluence, the adherent cells (fibroblasts like cells, bipolar cells) and unattached cells or floating cells (Schwann cell like cells) were pipetted out of the culture flask using EDTA- trypsin $(0.25 \%$ trypsin and $0.001 \mathrm{M}$ EDTA). The harvested cells were washed in PBS solution, and centrifuged at $1200 \mathrm{~g}$ for 2 minutes. The process of washing and centrifuging was repeated 3 times to remove any residual EDTA-trypsin from the cell cultures. Subsequently, the cells were subcultured and transferred onto a fresh $25 \mathrm{~cm}^{2}$ culture flasks containing DMEM with $10 \%$ foetal calf serum. This process of passage was repeated until the $4^{\text {th }}$ passage was achieved for the benign familial, plexiform and the sporadic NF1 specimens. The peripheral nerves from amputated stumps were utilized as comparative tissues and processed in a similar manner as done to the tumours.

\section{Phase II - Characterization of the Isolated Cells}

Following the $4^{\text {th }}$ passage, cell representatives from the culture flasks, containing the benign, plexiform and the sporadic NF1 were harvested from the flasks and subjected to cell characterization using cell markers for fibroblasts and Schwann cells (Vimentin and S-100 $\beta$ ) proteins, respectively. Briefly, $1.5 \times 10^{3}$ was counted with haemocytometer from each of the culture flasks containing the benign, plexiform and the sporadic NF1, and transferred onto a cell culture chamber slide (Invitrogen) and incubated for 3-5 days. Next, the culture medium was aspirated and the cells washed in PBS solution. The washed cells were then fixed in $10 \%$ methanol and air-dried at room temperature $\left(25^{\circ} \mathrm{C}\right)$ for 30 minutes.

Finally the air-dried and fixed cells were stained with commercially prepared primary antibodies mouse antiVimentin (cat \# 180052) and Rabbit-anti-S-100 $\beta$ (cat \#180046) Invitrogen for fibroblasts and Schwann cells respectively, and photomicrographs taken. 


\section{Phase III -Response to basic growth factor stimulus (bFGF)}

Following the cell characterization of the cell cultures, $0.1 \mathrm{ml}$ of bFGF was carefully introduced into the flasks containing $1.0 \times 10^{3}$ cells derived from experimental and control tissues. Cultures were allowed to grow at $37^{\circ} \mathrm{C}$ with $95 \% \mathrm{O}_{2}, 5 \% \mathrm{CO}_{2}$ and $100 \%$ humidity for $3-5$ days. Cultures were constantly monitored for growth and contamination with the aid of an inverted light microscope. Daily digital images of the culture were taken.

\section{Results}

The normal peripheral nerve did not show any appreciable growth advantage after the administration of the bFGF. The cell concentration dropped to $0.4 \times 10^{3} \mathrm{ml}$ on the $4^{\text {th }}$ day of the incubation as in shown in (Figure 1).

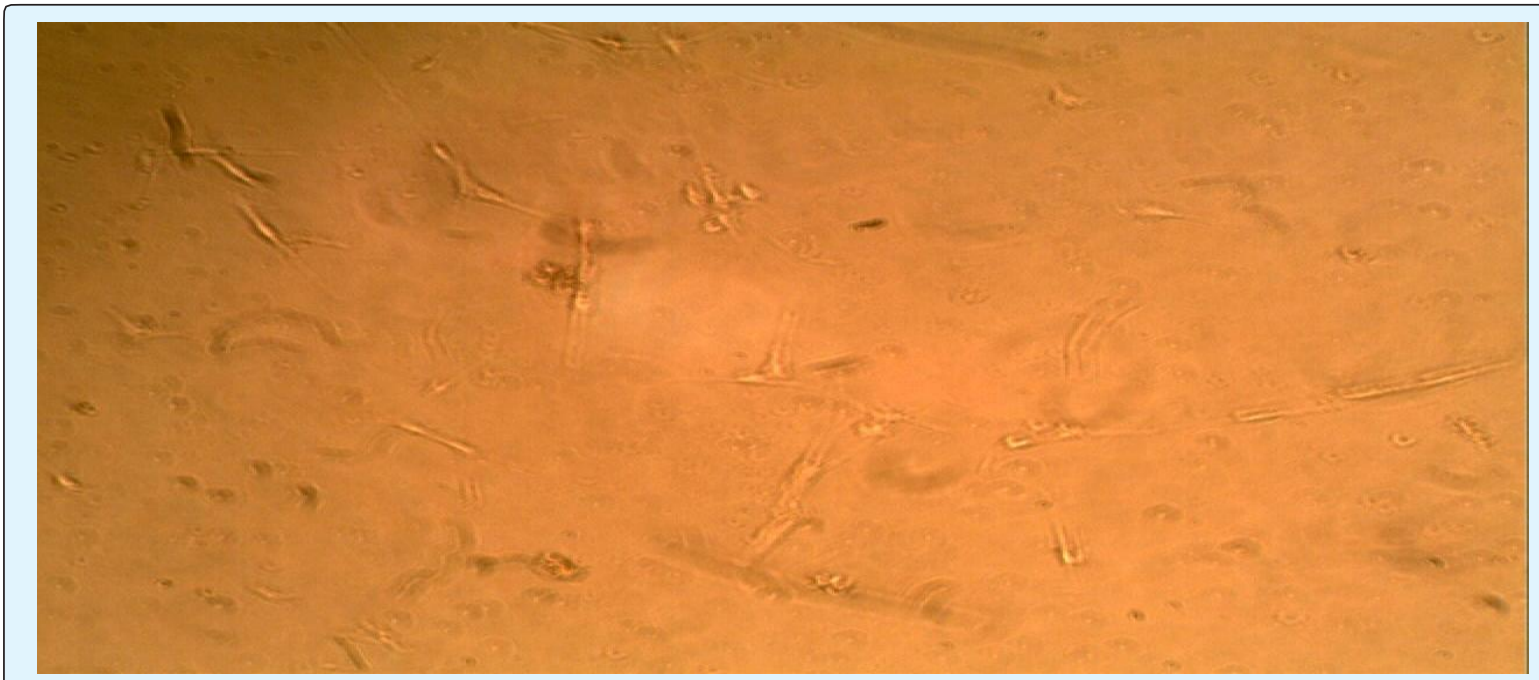

Figure 1: Normal peripheral nerves after treatment with bFGF exhibited a very sparse cell population $\left(0.4 \times 10^{3} / \mathrm{ml}\right)$ of largely bipolar cells (arrows) $(\mathrm{x} 200)$.

The benign familial neurofibroma exhibited a rather different growth pattern. The growth of the cells in this case was quite phenomenon. The cell population

increased from $1.0 \times 10^{3}$ to $4.6 \times 10^{3} / \mathrm{ml}$ after the $4^{\text {th }}$ passage (Figure 2).

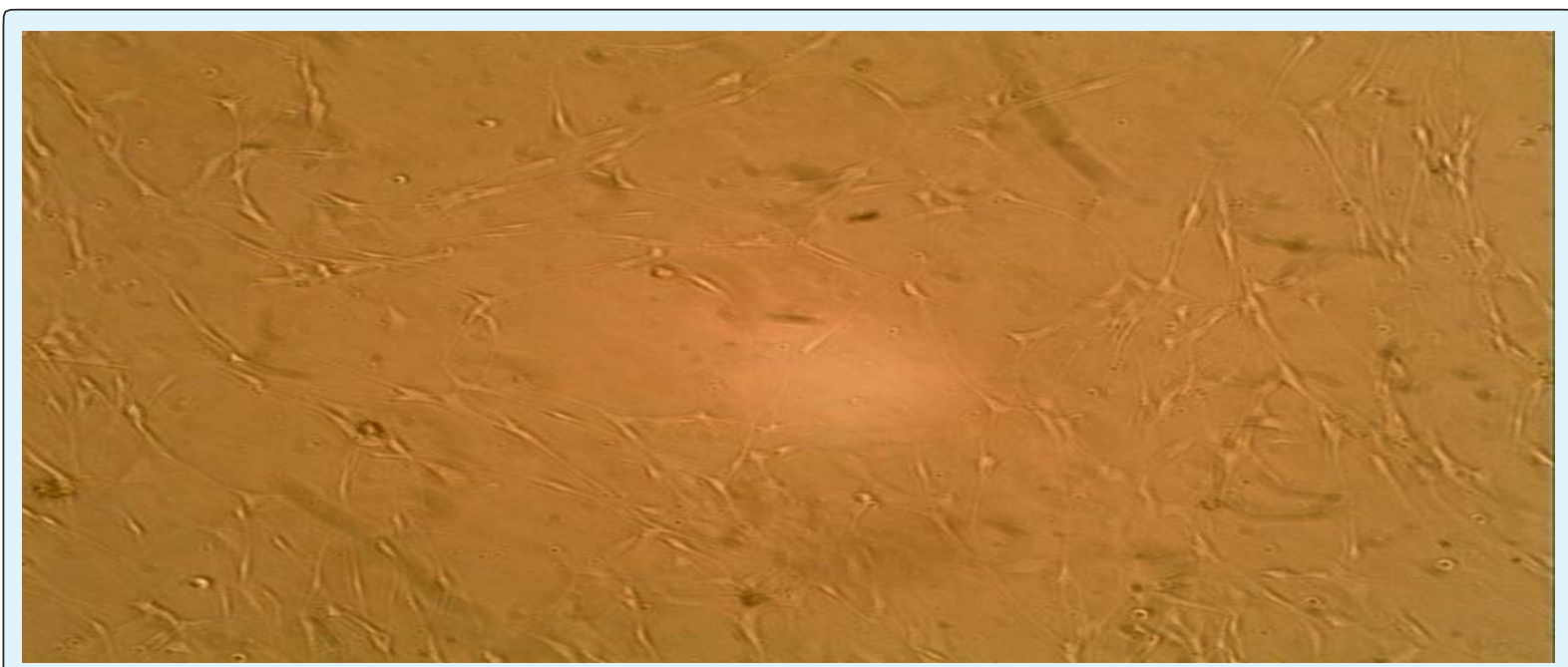

Figure 2: Benign familial neurofibroma cell culture $\left(4.60 \mathrm{x} 10^{3} / \mathrm{ml}\right)$ exhibiting dense cell population bipolar and tripolar cells (up and down arrows) that is close to being confluent after the addition of bFGF (x200). 
Cultures from the benign plexiform neurofibroma exhibited an overwhelming growth of the cells following the administration of $0.1 \mathrm{ml} b F G F$. The cell population was determined by haematocrit count to be $7.2 \times 10^{3} / \mathrm{ml}$ of the cell suspension as shown in (Figure 3).

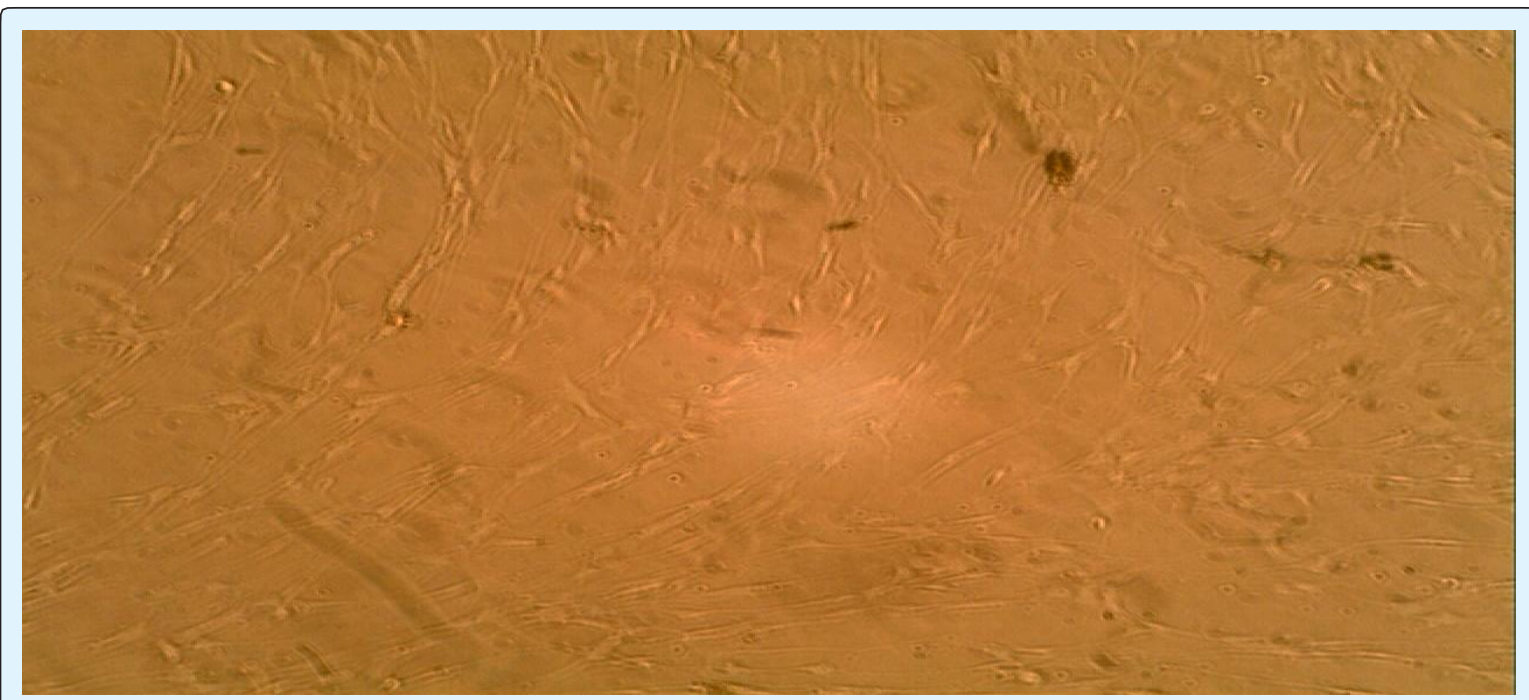

Figure 3: Plexiform neurofibroma cell culture showing an overwhelming cell proliferation of predominantly bipolar cells at confluent $\left(7.2 \mathrm{x} 10^{3} / \mathrm{ml}\right)$ after the treatment with basic fibroblast growth factor (bFGF) (x 100).

The sporadic neurofibromas demonstrated a cell count $\left(5.45 \times 10^{3} / \mathrm{ml}\right)$ after administration of the $0.1 \mathrm{ml}$ of bFGF as shown in (Figure 4). The pattern of cell growth in this sub-set of neurofibroma is similar to those obtained for the benign neurofibroma.

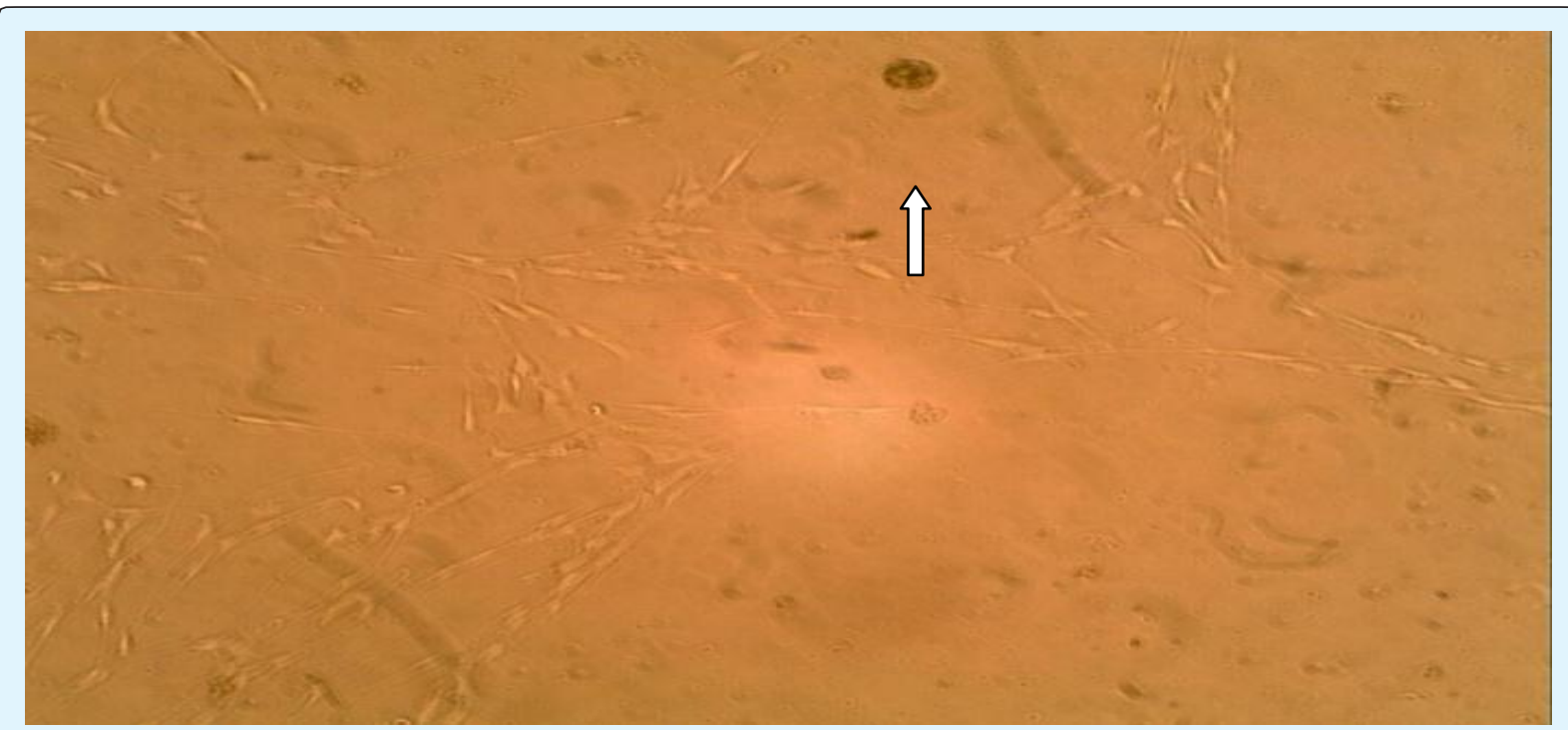

Figure 4: Sporadic neurofibroma cell culture at less than confluence $\left(1.45 \times 10^{3} / \mathrm{ml}\right)$ that is made up of largely 'cells with branching' (arrow) after treatment with bFGF (x 100). 


\section{Discussion}

No appreciable growth advantage was observed in the normal peripheral nerve following treatment with growth factor. This may be due to the assertion that the Schwann cells and fibroblasts, the major constituents of the normal peripheral nerve are held, in the GO \GI phase of the cell cycle and have differentiated. Though the Schwann cells have been suggested to have the inherent ability to "dedifferentiate" and assume a phenotype that allows it to proliferate, this phenomenon was only noticed in the pathologic tissues, but not in the normal peripheral nerves.

The somatic mutation of the cell culture was reported to be undetectable in fibroblast by the second passage, suggesting that this cell type may not be harbouring the 'second hit' [8]. On the contrary, somatic mutation was observed in a 98\% pure Schwann cell culture by the third passage [8]. The observations of Roudebush and coworkers is consistent with our study in which we observed that in both the familial and plexiform, the growth advantage was not appreciable after treatment with $10 \%$ foetal calf serum. This may be attributed to the NF1+/- status of the fibroblast in both the familial and plexiform neurofibroma and the NF1-/- status of the Schwann cells even though the plexiform subtypes can transform into malignant peripheral nerve sheath tumour (MPNST). This is more glaring in the sporadic neurofibroma, where the fibroblastic component of the sporadic neurofibroma do not harbour any loss of neurofibromin in any of the alleles $(\mathrm{NF}+/+)$. On the contrary, it is the Schwann cell (slow growing) component of the sporadic that harbours the defective allele (NF1-/-) and this may explain the sparse culture observed in the sporadic cell at the beginning in this study.

However, when bFGF was introduced into the culture plates, the normal peripheral nerve culture did not exhibit any significant growth as compared to when the bFGF was not added. We suggest that this finding may be due to the suggestion that the fibroblasts and the Schwann cells in the normal peripheral nerves have the full compliment $(\mathrm{NF} 1+/+)$ of the NF1 gene, and thus, the neurofibromin is able to reverse the active Ras (GTP) to inactive Ras (GDP). Hence, the cell proliferation is tightly controlled, and growth factors are denied access to the endoneurium of the peripheral nerve, where the Schwann cells are domicile. In support of this hypothesis, Aurrand-Lions, et al. reported that in 'endothelial cells with NF1+/- and
NF1-/- genotype, the cell-cell adhesion may be altered and the finely co-ordinated opening and closing of the endothelial cell-cell junctions is lost'. This would then lead to an unregulated access of growth factors into the endoneurium of the peripheral nerves [9].

Furthermore genetic studies have shown that NF1+/mice have increased angiogenesis in vivo and that disruption of neurofibromin in mouse endothelial cells could lead to cardiovascular defects $[5,10]$. In a similar fashion to the normal peripheral nerves, when the bFGF was added to the sporadic neurofibroma cell cultures, the growth was somewhat similar to those of the normal nerves. This result may be due to the fact that the fibrotic content of the sporadic neurofibroma have the full complement of the NF1 gene $(\mathrm{NF} 1+/+)$, while the Schwann cells that harbour the defective NF1 gene (NF1/-) and, thus, rate of the growth is quite slow. Conversely, we observed that when bFGF was introduced into the culture plates containing cells from plexiform and the familial neurofibromas, there was monumental increase in the rate of proliferation of the cultures. This may not be unconnected with the haploinsufficient NF1+/- gene status within the fibrotic constituents of the tumour in association with the NF-/- Schwann cells. Furthermore, this NF1 status of the fibroblasts and the Schwann cells may have provided an enabling environment for growth factor receptors to respond to the stimulus from the bFGF. In support of this notion, Kawachi, et al. have shown that NF1-/- Schwann cells secrete increased concentrations of vascular endothelial growth factor (VEGF) and bFGF [11]. These growth factors have been linked to the initiation of the so-called 'angiogenic switch', required for tumourigenesis. Furthermore, Munchhof, et al. have demonstrated that the NF1+/- mice have increased angiogenesis in response to NF1-/- Schwann cell-derived growth factors in vivo via the hyperactivation of the RasRaf-Mek-Erk pathway [12].

Importantly, in another study to demonstrate the role of NF1-/- Schwann in a heterozygous environment in tumourigenesis, using NF1 knock out model in mice, (containing NF1+/- Schwann cells and NF1+/- mast cell), no mast cell infiltration into the nerves was observed and the mice did not develop neurofibroma [12]. Similarly, in earlier studies using the tissue-specific knock-out model, NF1-/-Schwann cells and NF1+/+ mast cell also show no mast cell infiltration into peripheral nerves and these nerves fail to develop neurofibromas [13]. Conversely, (NF1-/- Schwann cells and NF1+/- mast cell) resulted in massive mast cell infiltration and develop plexiform 
neurofibromas in the mice [14]. The observations suggest the essential and critical role for the heterozygous environment in neurofibroma formation in vivo.

This study has demonstrated for the first time the critical role of nullizygous (NF1-/-) Schwann cell together with heterozygous (NF1+/-) fibrotic component of neurofibroma as essential for cell proliferation in vitro. Additionally, we hypothesized that for growth factor stimuli to reach the differentiated and quiescence Schwann cells, the tight junctions would have to be altered by the heterozygous tumor micro-environment.

\section{Conclusion}

This study has demonstrated that Schwann cells and fibroblast are the major cell constituent of neurofibroma and the normal peripheral nerve. The growth pattern of the normal peripheral nerve $(\mathrm{NF} 1+/+$ fibroblasts and Schwann cells) differs significantly from those of the tumours (NF1+\- fibroblasts, NF1-/- Schwann cells), when no growth factor was added to the culture medium. This may not be unconnected to the fact that the normal nerves do not harbour any NF1 mutant allele and, thus, the neurofibromin reverses the GTP active ras to the inactive state - GDP ras.

Normal nerve response to growth factor stimulus was observed to result in senescence and or apoptosis, perhaps due to the roles played by tumour suppressor genes such as s p53 and neurofibromin levels that would readily reverse the active ras to inactive form.

Conversely, the benign familial (NF1+/- fibroblast, NF1-/- Schwann cell) and sporadic (NF1+/+ fibroblast, NF1-/- Schwann cell) neurofibromas exhibited increase in proliferation following challenge with bFGF. Similarly, the plexiform neurofibromas (NF1+/- fibroblast, NF1-/Schwann cell), which are congenital and usually transform to MPNST exhibited the highest level of proliferation among the neurofibroma sub-types. Heterozygous environment is essential for cell proliferation in in-vitro study of neurofibroma.

\section{Acknowledgement}

We wish to acknowledge the invaluable assistance rendered by Prof S.P. Frostick, and Dr M.M. Roebuck of the University of Liverpool, UK. Further we are also grateful to Niger State Government of Nigeria and TET Fund for the financial support.

Bello A, et al. Growth Response of Neurofibroma Explants to FGF-Basic Human Single-Plex Beads In -Vitro Cell-Culture Analysis. J Human Anat 2017, 1(2): 000107.

\section{References}

1. Peltonen J, Jaakkola S, Lebwohl M, Renvall S, Risteli L (1988) Cellular differentiation and expression of matrix genes in type 1 Neurofibromatosis. Lab Invest 59(6): 760-777.

2. Korf BR (1999) Neurofibromas and Malignant Tumours of the Peripheral Nerve Sheath, In: Friedman JM, Gutmann DH, MacCollin M, Riccardi VM (Eds.), Neurofibromatosis: Phenotype, Natural History and Pathogenesis. Baltimore, John Hopkins Univ Press 142-161.

3. Muir DS, Varon MM (1990) Schwann cell proliferation in vitro under negative autocrine control. J Cell Biol 111: 2663-2671.

4. Muir D (1995) Differences in proliferation and invasion by normal, transformed and NF1- Schwann cell cultures are influenced by matrix metalloproteinase expression. Clin Exp Metast 13(4): 303-314.

5. Wu M, Wallace MR, Muir D (2005) Tumourigenic properties of neurofibromin-deficient Schwann cells in culture and as syngrafts in Nf1 knockout mice. J Neurosci Res 82(3): 357-367.

6. Wu J, Willams, JP, Rizvi TA, Kordich JJ, Witte D (2006) Plexiform and Dermal neurofibromas and Pigmentation are cause by NF1 loss in Desert Hedgehog-Expressing Cells. Cancer Cell 13(2): 105116.

7. Muir D, Neubauer D, Lim IT, Yachnis AT, Wallace MR (2001) Incidence of Neurofibromatosis Type -1. Am J Pathol 158: 501-513.

8. Roudebush M, Slabe T, Sundaram V, Hoppel CL, Golubic M, et al. (1997) Neurofibromin co-localizes with mitochondria in cultured cells. Exp Cell Res 236(1): 161-172.

9. Aurrand LM, Johnson LC, Wong C, Du P, Imhof BA (2001) Heterogeneity of endothelial junction is reflected by differential expression and subcellular localization of the three JAM family members. Blood 98(13): 3699-3707.

10. Gitler AD, ZhuY, Ismat FA, Lu MM, Yamauchi Y, et al. (2003) NF1 has an essential role in endothelial cells. Nat Genet 33(1): 75-79. 
11. Kawachi $Y, X u$ X, Ichikawa E, Imakado S, Otsuka F (2003) Expression of angiogenic factors in neurofibromas. Exp Dermatol 12(4): 412-417.

12. Munchhof AM, Li F, White HA, Mead LE, Krier TR, et al. (2006) Neurofibroma-associated growth factors activate a distinct signaling network to alter the function of neurofibromin-deficient endothelial cells. Hum Mol Genet 15(11): 1858-1869.

13. Parada LF, Kwon CH, Zhu Y (2005) Modeling neurofibromatosis type 1 tumors in the mouse for therapeutic intervention. Cold Spring Harb Symp Quant Biol 70: 173-176.

14. Dejana E (2004) Endothelial cell-cell junctions: Happy together. Nat Rev Mol Cell Biol 5: 261-270.

15. Nair S, Leung H, Collins A, Ramsden R, Wilson J (2007) Primary Cultures of Human Vestibular
Schwannoma: Selective Growth of Schwannoma Cells. Otol Neurol 28(2): 258-263.

16. Odebode TO, Afolayan EA, Adigun IA, Daramola 00 (2005) Clinopathological study of neurofibromatosis type 1; an experience in Nigeria. Int J Dermatol 44(2): 116-120.

17. Vreomen M, Weidner N (2003) Purification of Schwann cells by selection of p75 low affinity nerve growth factor receptor expressing cells from adult peripheral nerve. J Neurosci Methods 124(2): 135143.

18. Wu M, Wallace MR, Muir D (2001) Tumourigenic properties of neurofibromin-deficient neurofibroma Schwann cells. Am J Pathol 158(2): 501-513. 